\title{
A new era for TD, The Journal for Transdisciplinary Research in Southern Africa
}

With this edition TD The Journal for Transdisciplinary Research in Southern Africa celebrates its fifth anniversary. We extend a special word of thanks to our authors, editorial collaborators, and particularly to our readers for their support. It has been a period of interesting encounters. Asking colleagues to evaluate material submitted in a variety of fields - sometimes very distant from their own specialised areas of research - was a new venture for many. Some reviewers initially indicated that they were uncertain of the merits of this 'transboundary thing'. Subsequently, many of them experienced a change of heart. Others took the acceptance of transdisciplinarity in their stride. In the evaluations we received there was a sense of surprise; astonishment in the transformation taking place in the use and understanding of factual knowledge in their respective fields of specialisation. It has been an adventure for many of us. Hopefully the journey will continue to be one of intellectual exploration and discovery.

A periodical publication is like a living organism. It takes on unique personality traits, character and style that are representative of the thinking of those working to realise the objective of bringing out yet another edition. We like to think of TD as a vibrant medium of communication that is able to transform itself into a conduit for understanding the thoughts and ideas of scientists, educators and the broader spectrum of people in southern African society at any given time.

In many articles submitted, the theoretical underpinnings of transdisciplinarity were not a feature of discourse. Some mono-disciplinary articles we received were considered worthwhile publishing after peer reviewers advised us on the contribution and merits of the material. Although we will in future still consider such articles, there will be an increasing shift towards material that is either transdisciplinary, multi-disciplinary, or interdisciplinary in nature. This requires of authors to indicate in the textual discourse some aspects of transdisciplinary or multi-disciplinary research. There should be a theoretical underpinning that explains why the material is worthy of publication in TD. We must strive towards a broader discourse on scientific practice; this is currently too subdued. We need to bring it to the fore and become contemplative in the process of seeking answers to the scientific questions we pose - especially in the fields of theory and methodology.

We are open to suggestion on the publication of issues that focus on specific themes. Colleagues who have plans for interesting projects that can be organised as either a research initiative or a focused conference event, are welcome to communicate with us. We would like to see if and how we can assist in promoting interaction and the publication of current research.

We have been involved in discussions with researchers, writers and educators to determine if TD should introduce regular sub-sections that focus on research in certain fields of academic teaching. The objective is to determine how interactive, in terms of research collaboration, these areas have become. For example, one field that needs attention is the overall impact of information communications technologies (ICT) in all fields of science. Computers have become an integral part of our daily lives, not only as scientists and researchers, but also as ordinary people living out our lives in an ever-transforming society. Of particular 
importance is that some of the older generation of academics have tended to shun developments in information technology, or have failed to keep pace with new strides made in this direction. Although this is not always the product of their own doing, we should embark on a constructive search for options to determine how the apparent divide between human-machine interactions can be overcome.

There is also a need to integrate knowledge in the field of education. Why is it that some of the remarkable contemporary scientific discoveries are often overlooked by civil society? Could it be that important information is not properly communicated? This is reminiscent of a defunct culture associated with the ivory towers of the academe. In an interactive world of communication technology there should be some remedies for this short circuit in scientific knowledge dissemination. In southern Africa, a developing region of the world, it is important to be aware of breakthroughs and innovations in science. Although there are scientists and media practitioners in South Africa who are actively propagating better linkages with society at large, this should become an integral part of educators' teaching objectives at the primary, secondary and tertiary level. We have to communicate valuable information that speaks of now and anticipates tomorrow. We would like to think that TD can serve as a platform for education communication of this nature.

Writing science does not imply that it has to be written in a manner that is incomprehensible to a broader readership. Indeed, most good scientific material is written in a clear, unambiguous register. It has the greatest impact when the average intelligent person in society can understand it. The most gifted scientist is one who can state precisely in plain language what needs to be understood.

Speaking of language, the editorial policy of TD makes provision for authors to write in any of the 11 official languages of South Africa. With the exception of less than five articles in Afrikaans, all published material has been in English. We have not yet, for example, received a single scientific article written in isiXhosa, isiZulu, Sesotho, or Setswana. Publication of material that reflects the diversity of a regional society with a rich cultural heritage, a field still under-explored in our regional scientific writing, would justifiably make TD a proudly southern African publication.

In addition, we are open to publishing articles in international languages that have a substantial scientific readership. Here we think for example of Spanish, Portuguese, French, German, Chinese, Swahili and Arabic - languages also spoken in our region. If we are able to explore some of these communications we may be able to promote linkages with experts in other parts of the world. Given the possibility of using comprehensive summaries of articles, the discourse in a 'foreign' language need not exclude the majority of our readers. Instead it could pave the way for greater opportunities. For example, in the southern hemisphere, we share much in common with our continental neighbours in South America. Can we not use language as a vehicle to communicate ideas that are of mutual interest to our regional partners?

Finally, as of the next issue of TD, scheduled for July 2010, we will move into the digital era. Forthwith the journal will only be available in electronic format. The high costs associated with printing hardcopy editions, has forced us to move away from this valued medium of communication. We have a feeling of nostalgia and a sense of loss with the passing of the paper printing technology. On the brighter side, hopefully this change will inspire more readers to resort to comfortably settling in with electronic publications as a feature of an ever-transforming society. 